\title{
Associação entre o perfil lipídico e medidas antropométricas indicadoras de adiposidade em adolescentes
}

\author{
Association between lipids and anthropometric measures \\ of adiposity in adolescents
}

\author{
Neiva Leite \\ Fabrício Cieslak \\ Gerusa Eisfeld Milano \\ Suelen Meira Góes ${ }^{1}$ \\ Maria de Fátima Aguiar Lopes \\ Paulo César Barauce Bento ${ }^{1}$ \\ Ana Lúcia Campelo Prestes \\ Rosana Bento Radominski
}

\footnotetext{
${ }^{1}$ Universidade Federal do Paraná. Departamento de Educação Física. Núcleo de Pesquisa em Qualidade de Vida. Curitiba, PR, Brasil.
}

Recebido em 27/06/08 Revisado em 26/10/08 Aprovado em 01/11/08
Resumo - O objetivo do presente estudo foi analisar a associação entre os níveis lipídicos com a adiposidade global e central em adolescentes. Estudo transversal, descritivo e correlacional. Participaram 127 sujeitos púberes, 71 meninas (13,39+1,81 anos) e 56 meninos $(13,10 \pm 1,97$ anos). Verificou-se adiposidade por meio do índice de massa corporal (IMC) e a adiposidade central através da circunferência abdominal (CA), conforme idade, sexo e etnia. Determinou-se os níveis de colesterol total (CT), lipoproteína de alta densidade (HDL-C), lipoproteína de baixa densidade (LDL-C) e triglicérides (TG) após 12 horas de jejum. A análise de dados foi realizada pelo teste qui-quadrado e correlação de Pearson, considerando nível de significância de $\mathrm{p}<0,05$. Os meninos obesos apresentaram maiores proporções de HDL-C diminuído ( $\left.\aleph^{2}=11,036 ; p=0,004\right)$, LDL-C $\left(\aleph^{2}=8,283 ; p=0,016\right)$ e TG $\left(\aleph^{2}=6,422 ; p=0,04\right)$ aumentados em relação a não-obesos e com sobrepeso. Porém o CT foi similar entre os grupos. As meninas obesas apresentaram HDL-C menor comparado às meninas com sobrepeso e não-obesas $\left(\aleph^{2}=7,468 ; p=0,02\right)$. No entanto, as meninas nãoobesas, com sobrepeso e obesas não apresentaram proporções diferentes nas variáveis LDL$\mathrm{C}, \mathrm{TG}$ e CT $\left(\aleph^{2}=2,22 ; \mathrm{p}=0,259\right)$. Associações positivas foram verificadas para $\log n \mathrm{TG}$ e $\log n \mathrm{IMC}(\mathrm{r}=0,215 ; \mathrm{p}<0,001)$ e $\operatorname{logn} \mathrm{TG}$ e $\log n \mathrm{WC}(\mathrm{r}=0,210 ; \mathrm{p}<0,001)$, relações negativas foram observadas em $\log n$ HDL-C e $\log n$ IMC $(r=-0,425 ; \mathrm{p}<0,001)$ e $\log n$ TG e $\log n$ WC $(r=$ $0,365 ; \mathrm{p}<0,001)$. Neste estudo, houve maiores proporções de níveis diminuídos de HDL-C e aumentados de TG nos adolescentes obesos, comparado aos não-obesos. Observaram-se associações inversas moderadas do HDL-C com a adiposidade global e central.

Palavras-chave: Adiposidade; Adolescentes; Lipídios.

Abstract - The aim of this study was to determine the association between lipid levels and overall and central obesity in adolescents. A cross-sectional, descriptive correlation study. A total of 127 pubescent subjects participated in the study, 71 girls (13.39 \pm 1.81 years) and 56 boys (13.10 \pm 1.97 years). Overall adiposity was evaluated based on body mass index (BMI) and central adiposity was measured by waist circumference (WC) according to age, gender and race. Total cholesterol (TC), high-density lipoprotein (HDL-C), low-density lipoprotein (LDL-C) and triglycerides (TG) were determined after a 12-hour overnight fast. The results were analyzed using the chi-square test and Pearson's correlation, with the level of significance set at $\mathrm{p}<0.05$. Obese boys presented larger proportions of increased LDL-C $\left(\aleph^{2}=8.283 ; \mathrm{p}=0.016\right)$ and TG $\left(\aleph^{2}=6.422 ; \mathrm{p}=0.04\right)$ and reduced HDL-C $\left(\aleph^{2}=11.036\right.$; $\mathrm{p}=0.004)$ than non-obese and overweight adolescents. However, TC was similar among groups. Obese girls presented lower HDL-C levels than non-obese and overweight girls $\left(\aleph^{2}=7.468 ; p=0.02\right)$. No difference in LDL-C, TG or TC was observed between obese, overweight and non-obese girls. A positive association was observed between logn TG and $\operatorname{logn}$ BMI $(r=0.215 ; \mathrm{p}<0.001)$ and between $\operatorname{logn} \mathrm{TG}$ and $\operatorname{logn} \mathrm{WC}(\mathrm{r}=0.210 ; \mathrm{p}<0.001)$. A negative association was observed between logn HDL-C and logn BMI $(r=-0.425 ; \mathrm{p}<0.001)$ and between $\log \mathrm{TG}$ and $\log \mathrm{WC}(\mathrm{r}=-0.365 ; \mathrm{p}<0.001)$. This study demonstrated a higher proportion of reduced HDL-C levels and increased TG among obese adolescents compared to non-obese subjects. A moderate inverse association was observed between HDL-C and overall and central adiposity.

Key words: Adiposity; Adolescents; Lipids. 


\section{INTRODUÇÃO}

A obesidade aumentou em todas as faixas etárias da população e tem sido referida na literatura como um importante fator de risco para o desenvolvimento de doenças crônicas não-transmissíveis ${ }^{1,2}$. As doenças que antes eram encontradas apenas em adultos estão sendo cada vez mais diagnosticadas em crianças e adolescentes obesos, o que passou a ser uma preocupação para a Organização Mundial da Saúde, de forma que, aproximadamente, $80 \%$ das crianças obesas permanecem com excesso de peso na vida adulta ${ }^{3,4}$, possibilitando o desenvolvimento de diversos fatores de risco metabólicos e morte prematura ${ }^{5,6}$.

Sendo assim, os jovens que venham a apresentar fatores de risco com o avanço da idade, tendem a possuir maior predisposição para processos ateroscleróticos e, consequentemente, desenvolvimento de doenças cardiovasculares (DCV) ${ }^{7}$. A literatura relata que, em crianças e adolescentes obesos, as alterações metabólicas que se correlacionam positivamente com os riscos cardiovasculares mais frequentes são: as dislipidemias em 40 a 60\%, a resistência insulínica (RI) em 30 a 55\% $\%^{8,9}$, a intolerância à glicose em 20 a 25\% e o diabetes mellitus tipo 2 em $4 \%$ dos $\operatorname{casos}^{10}$.

Evidências indicam que a distribuição de gordura está relacionada com as DCV ${ }^{11,12}$. Freqüentemente demonstradas como fator independente do grau de obesidade, essas associações podem ser obtidas através dos diversos parâmetros antropométricos propostos, entre eles, as pregas cutâneas e circunferências corporais ${ }^{13}$, sendo procedimentos de fácil aplicabilidade e suficiente grau de acurácia ${ }^{14}$.

Os métodos mais utilizados para verificar o padrão corporal e a presença de fatores de risco cardiovasculares em crianças e adolescentes são o índice de massa corporal (IMC) ${ }^{15}$ e a circunferência abdominal $(\mathrm{CA})^{16}$. Entretanto, é visível a necessidade de estudos em populações pediátricas referentes à associação desses parâmetros antropométricos ao perfil lipídico de eutróficos e obesos, no sentido de que, os grandes escopos dessas evidências baseiam-se em dados internacionais. Desse modo, o presente estudo objetivou analisar a associação entre os níveis lipídicos com a adiposidade global e central em escolares curitibanos.

\section{PROCEDIMENTOS METODOLÓGICOS}

\section{Desenho e sujeitos da pesquisa}

O delineamento do estudo é qualificado como transversal, descritivo e correlacional. A coleta de dados foi realizada com pacientes da Unidade de Endocrinologia Pediátrica do Hospital de Clínicas (HC de Curitiba).

O cálculo de amostra foi efetuado através do programa Win Episcope (Win Epi, versão 2.0) e baseou-se em evidências prévias referentes à média e ao desvio padrão da pesquisa realizada por Sarni et al. ${ }^{1}$,com 65 sujeitos. O tamanho amostral mínimo foi de 32 indivíduos para cada grupo, em um nível de confiança de 0,05 , poder de 0,8 e magnitude de efeito moderado de 0,35 , conforme proposto por Cohen ${ }^{17}$. No entanto, houve dificuldade na obtenção dos exames de sangue dos indivíduos não-obesos e com sobrepeso.

Foram incluídos no estudo os sujeitos que: (a) se propuseram a efetuar todas as avaliações; (b) apresentaram o termo de livre consentimento assinado pelos responsáveis. Os critérios de exclusão foram: (a) indivíduos em fase pré-púbere; (b) sem utilização de medicamentos que interferissem no perfil lipídico.

○úmero de avaliados foi de 131 sujeitos, entretanto, 4 indivíduos foram excluídos do estudo pelo fato de possuírem classificação pré-púbere. Desse modo o tamanho amostral foi de 127 adolescentes, sendo 71 meninas $(13,39 \pm 1,77$ anos) e 56 meninos (13,05 $\pm 1,93$ anos). O protocolo de pesquisa foi aprovado pelo Comitê de Ética em Pesquisas do Setor de Ciências Biológicas da Universidade Federal do Paraná sob protocolo CEP/HC 765.184/200311 , conforme as normas estabelecidas na resolução 196/96 do Conselho Nacional de Saúde sobre pesquisa envolvendo seres humanos.

Medidas Antropométricas e Maturação Sexual As técnicas utilizadas para obtenção das medidas antropométricas foram realizadas conforme Lohman et $\mathrm{al}^{18}$. As medidas foram efetuadas pelo mesmo avaliador, com a realização de três medidas para consideração do valor médio entre as mesmas.

$\mathrm{Na}$ obtenção da estatura corporal (cm), utilizou-se um estadiômetro (Ayrton Corporation, precisão de $0,1 \mathrm{~cm}$ ) fixado à parede, sendo que o indivíduo deveria manter-se em posição ortostática, com os pés unidos, descalços, utilizando o mínimo possível de roupas. Além disso, deveria permanecer em apnéia respiratória e com a cabeça no plano horizontal de Frankfurt, tendo as superfícies posteriores do calcanhar, cintura pélvica, cintura escapular e região occipital em contato com o instrumento de medida.

A massa corporal $(\mathrm{kg})$ foi mensurada em balança (Filizola, precisão de $0,1 \mathrm{~kg}$ ) tipo plataforma, com 
o avaliado permanecendo em posição ortostática, no centro da plataforma, descalço e utilizando roupas íntimas. Em seguida, calculou-se o índice de massa corporal (IMC) mediante a utilização do índice de Quetelet, em $\mathrm{kg} / \mathrm{m}^{2}$ : IMC=massa corporal/estatura ${ }^{2}$. Para IMC, os indivíduos foram classificados segundo os critérios definidos por $\mathrm{CDC}^{19}$ para excesso de peso, de modo que, neste estudo, classificamos como obesos os indivíduos com o IMC entre o percentil $85^{\circ}$ e $95^{\circ}$ (sobrepeso) e acima do percentil $95^{\circ}$ (obeso), para baixo peso, no qual denominamos não-obesos os adolescentes abaixo do percentil $85^{\circ} \mathrm{e}$ acima do $5^{\circ}$ percentil. Ambos os métodos de ponto de corte foram utilizados conforme sexo, idade e etnia.

Para determinação da CA, foi utilizada uma fita antropométrica inelástica (precisão de $0,1 \mathrm{~cm}$ ), aplicada no ponto médio entre a crista ilíaca e a face externa da última costela, paralela ao solo, com o sujeito em pé, com o abdômen relaxado, os braços ao longo do corpo e os pés unidos. A classificação da circunferência abdominal (CA) foi baseada nos critérios propostos por Fernandez et $\mathrm{al}^{16}$, que consideram os valores acima ou iguais ao $75^{\circ}$ percentil, como limítrofes ou aumentados, conforme idade, sexo e etnia.

A avaliação puberal dos indivíduos foi realizada pela auto-avaliação através de gravuras conforme os estágios maturacionais propostos por Tanner ${ }^{20}$, sendo que, as meninas foram avaliadas quanto ao desenvolvimento mamário e a pilificação pubiana e os meninos foram mensurados quanto ao tamanho testicular e a pilificação pubiana.

\section{Exames Laboratoriais}

As amostras sanguíneas foram coletadas no período da manhã, após 12 horas de jejum, para realização de hemograma e dosagens de glicose, insulina, colesterol total (CT), lipoproteína de alta densidade (HDL-C), lipoproteína de baixa densidade (LDL-C) e triglicérides (TG). As mensurações foram realizadas nas sessões de Bioquímica, Hematologia e Dosagens Hormonais do Serviço de Análises Clínicas do HC da Universidade Federal do Paraná (UFPR).

As concentrações plasmáticas de CT, TG e de HDL-C foram determinadas em mg/dl, através de teste colorimétrico enzimático (CHOD-PAP) (Laboratório Merck, Darmstadt, Alemanha; Laboratório Roche, Indianápolis, IN, EUA). O LDL-colesterol foi calculado pela equação de Friedewald $^{21}$, em mg/dl: LDL $=\mathrm{CT}-(\mathrm{HDL}+\mathrm{TG} / 5)$. Considerou-se como referência os valores para lipídios apresentados na I Diretriz de Prevenção da Aterosclerose na Infância e na Adolescência ${ }^{22} \mathrm{com}$ exceção para os níveis de HDL-C, que foram considerados os valores maiores ou iguais a $45 \mathrm{mg} / \mathrm{dl}$.

\section{Classificação dos Exames Laboratoriais}

Consideraram-se como referência os valores para lipídios apresentados na I Diretriz de Prevenção da Aterosclerose na Infância e na Adolescência ${ }^{22}$. Foram consideradas alteradas as avaliações de CT que apresentaram valores superiores a $170 \mathrm{mg} / \mathrm{dL}$, de LDL-C e TG superior a $130 \mathrm{mg} / \mathrm{dL}$ e HDL-C inferior a $45 \mathrm{mg} / \mathrm{dL}$.

\section{Análise Estatística}

Utilizaram-se medidas descritivas, tabelas e gráficos para caracterização dos participantes. Para comparação de proporções entre adolescentes obesos e não-obesos, foi realizado o teste qui-quadrado. A normalidade dos dados foi realizada pelo teste de Shapiro-Wilk, em seguida os dados foram transformados em valores de logaritmo natural $(\log n)$ para normalização, após este procedimento aplicaram-se o teste $T$ independente, na comparação das variáveis entre os sexos, e a correlação de Pearson, para analisar as possíveis relações do IMC e CA com as mensurações lipídicas. Os procedimentos estatísticos do presente estudo foram realizados mediante a utilização do Statistical Package for the Social Sciences (SPSS, versão 13.0) for Windows, para um nível de significância fixado em $\mathrm{p}<0,05$.

\section{RESULTADOS}

As características descritivas de todos os sujeitos do estudo, separados por sexo, são apresentadas na Tabela 1. Os resultados demonstram que as meninas apresentam maiores valores em relação aos meninos apenas no IMC $(p=0,02)$ e não apresentaram diferenças significativas na idade $(\mathrm{p}=0,313)$, massa corporal $(p=0,161)$, estatura $(p=0,063), C A$ $(\mathrm{p}=0,33), \mathrm{CT}(\mathrm{p}=0,858)$, HDL-C $(\mathrm{p}=0,768)$, LDL-C $(\mathrm{p}=0,904)$ e TG $(\mathrm{p}=0,831)$.

A figura 1 mostra as comparações de variáveis lipídicas em meninas. As proporções indicam que as meninas obesas apresentaram HDL-C significativamente menor, comparado às meninas com sobrepeso e não-obesas $\left(\aleph^{2}=7,468 ; p=0,02\right)$. No entanto, as meninas não-obesas, com sobrepeso e obesas não apresentaram proporções diferentes nas variáveis LDL-C $\left(\aleph^{2}=3,579 ; \mathrm{p}=0,167\right)$, TG $\left(\aleph^{2}=2,697 ; \mathrm{p}=0,259\right)$ e CT $\left(\aleph^{2}=2,22 ; \mathrm{p}=0,259\right)$. 
Tabela 1. Comparação das médias de tratamento por sexo, pelo teste t independente, para as variáveis antropométricas e lipídicas do grupo estudado.

\begin{tabular}{lcc}
\hline & Meninas $(\mathrm{N}=71)$ & Meninos $(\mathrm{N}=56)$ \\
\hline Idade (anos) & $13,39 \pm 1,77$ & $13,05 \pm 1,93$ \\
Estatura $(\mathrm{cm})$ & $157,83 \pm 8,68$ & $161,20 \pm 11,99$ \\
Massa Corporal $(\mathrm{kg})$ & $72,79 \pm 21,40$ & $67,89 \pm 16,66$ \\
IMC $\left(\mathrm{kg} / \mathrm{m}^{2}\right)$ & $28,78 \pm 6,80^{*}$ & $26,11 \pm 5,68$ \\
CA $(\mathrm{cm})$ & $92,75 \pm 18,26$ & $89,73 \pm 15,84$ \\
CT $(\mathrm{mg} / \mathrm{dL})$ & $160,32 \pm 32,71$ & $161,35 \pm 31,80$ \\
HDL-C $(\mathrm{mg} / \mathrm{dL})$ & $45,40 \pm 9,93$ & $45,94 \pm 10,48$ \\
LDL-C (mg/dL) & $94,21 \pm 29,57$ & $94,83 \pm 28,18$ \\
TG $(\mathrm{mg} / \mathrm{dL})$ & $107,29 \pm 62,70$ & $109,89 \pm 74,19$ \\
\hline
\end{tabular}

"*” indica diferença significante para $p<0,05$.

Tabela 2. Correlações de IMC e CA entre as variáveis lipídicas das meninas.

\begin{tabular}{|c|c|c|c|c|c|c|}
\hline Meninas & Logn & Logn & Logn & Logn & Logn & Logn \\
\hline$(\mathrm{N}=71)$ & IMC & CA & CT & HDL-C & LDL-C & TG \\
\hline Logn IMC & 1 & & & & & \\
\hline $\operatorname{Logn} C A$ & $0,973^{* *}$ & 1 & & & & \\
\hline Logn CT & 0,004 & 0,000 & 1 & & & \\
\hline Logn LDL-C & 0,086 & 0,067 & $0,900^{* *}$ & $-0,007$ & 1 & \\
\hline Logn TG & 0,192 & 0,219 & $0,370^{* *}$ & $-0,276^{* *}$ & 0,147 & 1 \\
\hline
\end{tabular}

“**" indica diferença significante para $p<0,01 ;$ " ** indica diferença significante para $p<0,05$.

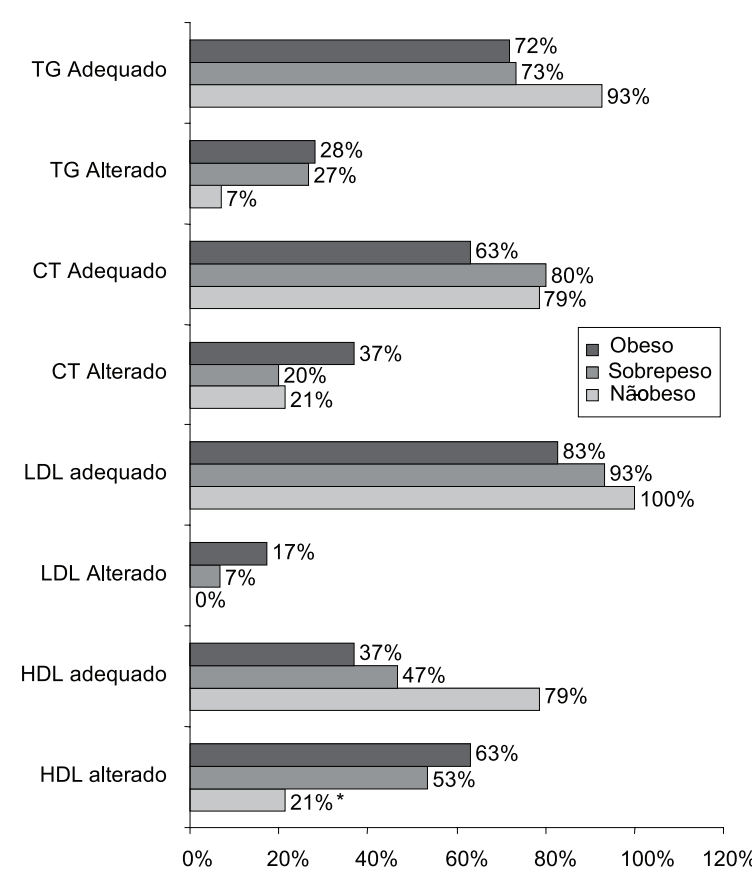

Figura 1. Proporções das variáveis lipídicas para as meninas.

Na Tabela 2, aparecem os valores de correlações de IMC, CA e perfil lipídico nas meninas. Houve correlação negativa do HDL-C com o IMC

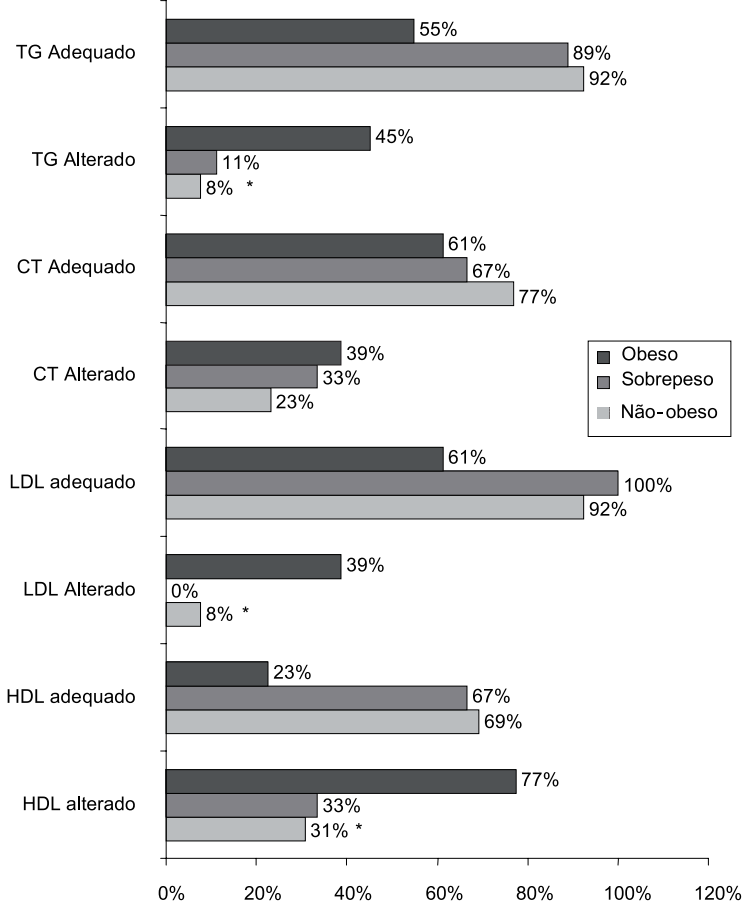

Figura 2. Proporções das variáveis lipídicas para os meninos. $(\mathrm{r}=-0,473 ; \mathrm{p}=0,001)$ e CA $(\mathrm{r}=-0,457 ; \mathrm{p}=0,001) . \mathrm{O}$ CT apresentou relação com o LDL-C (r=0,900; 
Tabela 3. Correlações de CA e variáveis metabólicas dos meninos.

\begin{tabular}{|c|c|c|c|c|c|c|}
\hline Meninos & Logn & Logn & Logn & Logn & Logn & Logn \\
\hline$(N=56)$ & IMC & $\mathrm{CA}$ & CT & HDL-C & LDL-C & $\mathrm{TG}$ \\
\hline Logn IMC & 1 & & & & & \\
\hline Logn CA & $0,951 * *$ & 1 & & & & \\
\hline Logn CT & $-0,036$ & $-0,061$ & 1 & & & \\
\hline Logn LDL-C & $-0,026$ & $-0,044$ & $0,925^{* *}$ & $-0,035$ & 1 & \\
\hline Logn TG & $0,266^{*}$ & $0,264^{*}$ & 0,219 & $-0,460^{* *}$ & 0,109 & 1 \\
\hline
\end{tabular}

"**" indica diferença significante para $\mathrm{p}<0,01$; "*" indica diferença significante para $\mathrm{p}<0,05$.

$\mathrm{p}=0,001)$ e $\mathrm{TG}(\mathrm{r}=0,370 ; \mathrm{p}=0,001)$. O HDL-C apresentou relação negativa com os $\mathrm{TG}(\mathrm{r}=-0,276$; $\mathrm{p}=0,023$ ).

Na figura 2, são apresentadas as comparações de meninos obesos, com sobrepeso e não-obesos para as variáveis lipídicas. Os meninos obesos apresentaram maiores proporções de HDL-C diminuído $\left(\aleph^{2}=11,036 ; \mathrm{p}=0,004\right)$, LDL-C $\left(\aleph^{2}=8,283\right.$; $\mathrm{p}=0,016)$ e TG $\left(\aleph^{2}=6,422 ; \mathrm{p}=0,04\right)$ aumentados em relação aos meninos não-obesos e com sobrepeso. Os resultados indicaram valores similares entre os grupos para CT $\left(\aleph^{2}=0,999 ; \mathrm{p}=0,606\right)$.

Os valores das correlações entre IMC, CA e as variáveis do perfil lipídico dos meninos estão na Tabela 3. O IMC demonstrou interação negativa com o HDL-C $(r=-0,378 ; p=0,004)$ e positiva com os TG $(r=0,266 ; p=0,047)$, do mesmo modo, a CA apresentou relações similares com o HDL- $\mathrm{C}(\mathrm{r}=$ $0,363 ; p=0,006)$ e TG $(r=0,264 ; p=0,04)$. O CT apresentou relação positiva com o LDL-C $(r=0,925$; $\mathrm{p}=0,001$ ), além disso, o HDL-C mostrou relação negativa com os TG $(r=-0,460 ; p=0,001)$.

\section{DISCUSSÃO}

As DCV são as causas mais frequentes de morbidade e mortalidade ${ }^{6}$, no sentido de que este processo decorre a partir da infância e adolescência, quando excesso de peso e a distribuição da gordura corporal elevam a possibilidade de alterações no perfil metabólico e complicações cardiovasculares em idades precoce ${ }^{7,23}$, principalmente, na presença da obesidade visceral ${ }^{24}$. Neste estudo, avaliaram-se as relações entre as medidas antropométricas e o perfil lipídico de adolescentes. As diferenças na idade cronológica dos participantes foram minimizadas pela avaliação puberal, em que foram incluídos no estudo somente os indivíduos classificados como púberes.

Estudos referentes ao perfil de distribuição de gordura entre crianças e adolescentes possuem re- sultados, demonstrando que IMC e CA apesar de serem medidas indiretas, são excelentes formas de avaliação da adiposidade global ${ }^{18}$ e central ${ }^{24}$, sendo associadas com o aumento dos marcadores metabólicos de resistência insulínica ${ }^{25}$. Quanto mais elevados forem IMC e CA, maior é a probabilidade em apresentar hipertensão arterial, hipertrigliceridemia, hipercolesterolemia e redução do HDL-C ${ }^{4,13}$.

No estudo realizado por Savva et $\mathrm{al}^{26}$,em uma amostra de crianças cipriotas de 10 a 14 anos, foi verificado que a CA é um bom preditor de DCV em comparação ao IMC, de forma que, as crianças acima do $75^{\circ}$ percentil apresentaram maiores taxas de CT, LDL-C e TG. No estudo de Lee et $\mathrm{a}^{24} \mathrm{com}$ uma amostra bi-racial de adolescentes e conforme pontos de corte proposto por Fernandez et $\mathrm{al}^{16}$, foi observado que com o aumento das categorias dos percentis de CA houve maiores níveis de TG e HDL-C. Os resultados do nosso estudo demonstram que os valores lipídicos estão associados similarmente com as variáveis antropométricas para os dois sexos, evidenciando que tanto IMC como CA são ferramentas de suma importância para o diagnóstico de DCV.

O estudo de Boyd et $\mathrm{al}^{27}$ avaliou o perfil lipídico em 497 crianças, entre 2 a 18 anos, com excesso de peso e observou que os meninos severamente obesos apresentaram níveis de HDL-C reduzidos em maior proporção do que os moderadamente obesos. No entanto, os meninos moderadamente obesos tiveram maiores proporções nos níveis de CT e LDL-C aumentados, em comparação aos severamente obesos. Entre as meninas, houve diferença significativa somente para os níveis de TG aumentados nas moderadamente obesas. Um fator limitante é que esses autores classificaram as variáveis lipídicas conforme os critérios para a população adulta. Portanto, foi possível comparar com os nossos resultados apenas as categorizações de HDL-C e LDL-C. Os resultados encontrados 
em nosso estudo mostram que tanto as meninas $\left(\aleph^{2}=7,47 ; p=0,006\right)$ quanto os meninos $\left(\aleph^{2}=8,61 ;\right.$ $\mathrm{p}=0,003)$ obesos apresentaram menores valores para HDL-C e que somente os meninos apresentaram maiores valores para os níveis de TG, quando comparados com o grupo dos eutróficos $\left(\aleph^{2}=4,61\right.$; $\mathrm{p}=0,03)$ e de LDL $\left(\aleph^{2}=4,23 ; \mathrm{p}=0,04\right)$. Ambos os sexos não apresentaram diferenças de CT e no LDL-C apenas as meninas não diferiram.

No estudo de Lee et $\mathrm{al}^{24}$, observou-se que a CA mostrou correlação significativa com TG e HDL-C. Maffeis et $\mathrm{al}^{14}$, encontraram correlação entre CA e HDL-C, não apresentando correlação com LDL-C. No presente estudo, para os meninos, observou-se correlação positiva da CA e IMC com os TG e negativa com o HDL-C, entretanto, para as meninas foi identificada apenas relação para o HDL-C. O CT apresentou relação com o LDL-C em ambos os sexos e com TG somente nas meninas. O HDL-C apresentou relação negativa com os TG nos dois grupos.

Poucos estudos avaliaram a relação entre variáveis antropométricas e fatores de risco cardiovascular em crianças 4,25,26,28. Em geral, os estudos não encontraram fortes correlações entre os métodos avaliativos de adiposidade global e central com os níveis de CT e de LDL-C em adolescentes ${ }^{23,25}$. Nesta pesquisa, foram encontradas correlações significativas da CA e IMC com os níveis de HDL-Ce TG (apenas para os meninos). Além disso, as duas medidas indiretas tiveram uma associação consistente com fatores de risco cardiovasculares como em outros estudos ${ }^{13,14}$, desse modo, caracterizando a CA como instrumento adequado para avaliação de co-morbidades ${ }^{14,15,16,24,26}$.

Nossa pesquisa deve ser analisada diante de algumas limitações como: ausência de avaliação dos hábitos alimentares dos adolescentes do estudo, fato que pode ser conflitante devido à maior ingestão de alimentos ricos em gordura total e saturada e que pode interferir nas concentrações plasmáticas de lipídios-lipoproteínas, ${ }^{7,29}$, o mesmo ocorrendo com o uso do álcool ${ }^{27}$ e tabaco ${ }^{7,29}$ que não foram avaliados. O delineamento amostral de seleção não-aleatória dos sujeitos com características étnicas ${ }^{12,27}$ próprias pode ter ocasionado um viés de seleção, dificultando a validade externa do estudo e a generalização dos resultados ${ }^{27,29}$.

Com base nestas implicações, sugerimos que os pediatras e outros profissionais da área da saúde, além de incentivar hábitos de vida saudáveis, devam incluir medidas antropométricas simples em suas avaliações clínicas. Desta forma, as suas mensurações poderão ser utilizadas como medidas diagnósticas para rastreamento da obesidade e o maior risco de dislipidemias, principalmente, aumento dos TG e redução do HDL-C em crianças e adolescentes. A avaliação do perfil lipídico em indivíduos que apresentem obesidade global e central minimizará o efeito deletério, em médio e longo prazo, desses fatores de risco sobre a saúde endotelial, à medida que possibilite o diagnóstico precoce das dislipidemias na população pediátrica, resultando em atitudes preventivas e terapêuticas para um estilo de vida adequado.

\section{CONCLUSÃO}

Neste estudo, foi observada correlação moderada e positiva entre a CA com o IMC e também com os TG. Houve correlação inversa entre a CA e os níveis de HDL-C nos adolescentes estudados. Evidenciando que ambos os instrumentos indiretos são preditores independentes de DCV. Estudos prospectivos com uma série de mensurações da CA, IMC e perfil metabólico são necessários para confirmar nossos achados. Necessitando, ainda, definir claramente os pontos de corte para a CA em adolescentes para classificá-los com aumento nos riscos à saúde devido ao excesso de gordura abdominal. Neste sentido, a inclusão da avaliação da CA nas diretrizes de síndrome metabólica pediátrica pode auxiliar na determinação dos fatores de riscos à saúde.

O estímulo às mudanças no comportamento, buscando a alimentação mais saudável e a prática regular de atividades físicas faz parte das ações preventivas e terapêuticas das doenças cardiovasculares na população jovem.

\section{REFERENCIAS BIBLIOGRÁFICAS}

1. Sarni RS, Souza FIS, Schoeps DO, Catherino P, Oliveira MACP, Pessoti CFX, et al. Relação da cintura abdominal com a condição nutricional, perfil lipídico e pressão arterial em pré-escolares de baixo estrato socioeconômico. Arq Bras Cardiol 2006;87(2):153-158.

2. Wang Y, Monteiro CA, Popkin BM. Trends of obesity and underweight in older children and adolescents in the United States, Brazil, China and Russia. Am J Clin Nutr 2002;75(6):971-977.

3. Janssen I, Katzmarzyk P, Boyce C, Vereecken C, Mulvihill C, Roberts C, et al. Comparison of overweight and obesity prevalence in school-aged youth from 34 countries and their relationships with physical activity and dietery patterns. Obes Rev 2005;6(2):123-132.

4. Freedman DS, Khan LK, Dietz WH, Srinivasan SR, Berenson GS. Relationship of childhood obesity to coronary heart disease risk factors in adulthood: The 
Bogalusa Heart Study. Pediatrics 2001;108(3):712-718.

5. Lissau I, Overpeck MD, Ruan WJ, Due P, Holstein $\mathrm{BE}$, Hediger ML. Body mass index and overweight in adolescents in 13 European Countries, Israel, and the United States. Arch Pedia and Adolesc Med 2004;158(1):27-33.

6. Lakka HM, Laaksonen DE, Lakka TA et al. The metabolic syndrome and total and cardiovascular disease mortality in middle-aged men. JAMA 2002; 288(21): 2709-2716.

7. Guedes DP, Guedes JERP, Barbosa DS, Oliveira JA, Stanganelli LCR. Fatores de risco cardiovasculares em adolescentes: indicadores biológicos e comportamentais. Arq Bras Cardiol 2006;86(6):439-450.

8. Lima SCVC, Arrais RF, Almeida MG, Souza ZM, Pedrosa LFC. Plasma lipid profile and lipid peroxidation in overweight or obese children and adolescents. J Pediatr 2004;80(1):23-28.

9. Sullivan CS, Beste J, Cummings DM, Hester VH, Holbrook T, Kolasa KM, et al. Prevalence of hyperinsulinemia and clinical correlates in overweight children reffered to lifesytle intervention. J Am Diet Assoc 2004;104(3):433-436

10. Sinha R, Fisch G, Teague B, Tamborlane WV, Banyas B, Allen K, et al. Prevalence of impaired glucose tolerance among children and adolescents with marked obesity. N Engl J Med 2002;346(11):802-810.

11. Daniels SR, Morrison JA, Sprecher DL, Khoury P, Kimball TR. Association of body fat distribution and cardiovascular risk factors in children and adolescents. Circulation 1999;99(4):541-545.

12. Goran MI, Gower BA. Relation between visceral fat and disease risk in children and adolescents. Am J Clin Nutr 1999;70(1):149-156.

13. Freedman DS, Serdula MK, Srinivasan SR, Berenson GS. Relation of circumferences and skinfold thickness to lipid and insulin concentrations in children and adolescents: the Bogalusa Heart Study. Am J Clin Nutr 1999;69(2):308-317.

14. Maffeis C, Pietrobelli A, Grezzani A, Provera S, Tato L. Waist Circumference and Cardiovascular Risk Factors in Prepubertal Children. Obes Res 2001;9(3):179-187.

15. Katzmarzyk PT, Srinivasan SR, Chen W, Malina RM, Bouchard C, Berenson GS. Body mass index, waist circumference, and clustering of cardiovascular disease risk factors in a biracial sample of children an adolescents. Pediatrics 2004;114(2):198-205.

16. Fernandez JR, Redden DT, Petrobelli A, Allisson DB. Waist circumference percentiles in nationally representative samples of african-american, european-american, and mexican-american, children and adolescents. J Pediatr 2004;145(4):439-444.

17. Cohen J. Statistical power analysis for the behavioral sciences. New Jersey: Erlbaum, 1988.

18. Lohman TG, Roche AF, Martorel R. Anthropometrics standardization reference manual. Ilinois: Human Kinetics, 1988.
19. Kuezmarski RJ, Ogden CL, Grummer-Estrawn LM, Flegal KM, Guo SS, Wei R, Mei Z, Curtin LR, Roche AF, Johnson CL. CDC growth charts: United States. Adv Data 2000:1-27.

20. Tanner JM. Normal growth and techniques of growth assessment. Clin Endocrinol Metab 1986;15(3):411-451.

21. Friedewald WT, Levy RI, Fredrickson DS. Estimation of the concentration of low-density lipoprotein cholesterol in plasma, without use of the preparative ultracentrifugue. Clin Chem 1972;18(6):499-502.

22. Sociedade Brasileira de Cardiologia. I Diretriz de prevenção da aterosclerose na infância e adolescência. Arq Bras Cardiol 2005;85(supl VI):1-36.

23. Thomas N-E, Cooper S-M, Williams SP, Baker JS, Davies B. Relationship of Fitness, Fatness, and CoronaryHeart-Disease Risk Factors in 12- to 13-Year-Olds. Pediatr Exerc Sci 2007;19(1):93-101.

24. Lee S, Bacha F, Arlanian S A. Waist circumference, blood pressure, and lipid components of the metabolic syndrome. J Pediatr 2006;149(6):809-816.

25. Botton J, Heude B, Kettaneh A, Borys JM, Lommez A, Bresson JL. Cardiovascular risk factor levels and their relationships with overweight and fat distribution in children: the fleurbaix laventie ville santé II study. Metabolism Clinical and Experimental 2007;56(5):614-622.

26. Savva SC, Tornaritis M, Savva ME, Kourides Y, Panagi A, Silikiotou N, et al. Waist circumference and waistto-height ratio are better predictors of cardiovascular disease risk factors in children than body mass index. Int J Obes Relat Metab Disord 2000;24(11):1453-1458.

27. Boyd GS, Koenigsberg J, Falkner B, Gidding S, Hassink S. Effect of Obesity and High Blood Pressure on Plasma Lipid Levels in Children and Adolescents. Pediatrics 2005;116(2):442-446.

28. Chu NF, Rimm EB, Wang DJ, Liou HS, Shieh SM. Relationship between anthropometric variables and lipid levels among school children: the Taipei Children Hearth Study. Int J Obes Relat Metab Disord 1998;22(1):66-72.

29. Guedes DP, Guedes JERP, Barbosa DS, Oliveira JA. Dispêndio energético diário e níveis de lipídeoslipoproteínas plasmáticos em adolescentes. Rev Bras Med Esp 2007;13(2):123-128.
Endereço para correspondência
Neiva Leite
Departamento de Educação Física, Universidade
Federal do Paraná.
Rua Coração de Maria, 92 (BR-116, km 92).
Jardim Botânico
CEP 80215-370 - Curitiba, Paraná, Brasil
E-mail: neivaleite@gmail.com 